\title{
Analytical Solutions of Dynamic Crack Models of Bridging Fiber Pull-Out in Unidirectional Composite Materials
}

\author{
Yuntao Wang', Yunhong Cheng ${ }^{2}$, Nianchun L $\ddot{u ̈}^{3}$, Jin Cheng ${ }^{4}$ \\ ${ }^{1}$ College of Mechanical Engineering, Liaoning Technical University, Fuxin, China \\ ${ }^{2}$ Department of Civil Engineering, Northeastern University, Shenyang, China \\ ${ }^{3}$ School of Material Science and Engineering, Shenyang Ligong University, Shenyang, China \\ ${ }^{4}$ Department of Astronautics and Mechanics, Harbin Institute of Technology, Harbin, China \\ Email: wyt234@163.com,cyh_neu@163.com,1nc_65@163.com,chengjin@ hit.edu.cn
}

Received September 22, 2013; revised October 29, 2013; accepted November 15, 2013

Copyright (C) 2013 Yuntao Wang et al. This is an open access article distributed under the Creative Commons Attribution License, which permits unrestricted use, distribution, and reproduction in any medium, provided the original work is properly cited.

\begin{abstract}
An elastic analysis of an internal central crack with bridging fibers parallel to the free surface in an infinite orthotropic anisotropic elastic plane was analyzed, and the crack extension should occur in the format of self-similarity. When the fiber strength is over its maximum tensile stress, the fiber breaks. By means of complex variable functions, the problem considered can be easily translated into Reimann-Hilbert mixed boundary value problem. Utilizing the built dynamic model of bridging fiber pull-out in unidirectional composite materials, analytical solutions of the displacements, stresses and stress intensity factors under the action of increasing loads $P t^{5} / x^{5}, P x^{5} / t^{4}$ are obtained, respectively. After those analytical solutions were used by superposition theorem, the solutions to arbitrary complex problems were acquired.
\end{abstract}

Keywords: Composite Materials; Bridging Fibers; Analytical Solutions; Crack; Variable Loads

\section{Introduction}

It is well known that the matrix cracking as well as fracture process of the bridging fibers is one of the significant mechanisms of the cracking expansion in fiberreinforced composite materials, such as unidirectional fiberreinforced brittle matrix composites [1,2], and threedimensional fiber-reinforced composites with an orthogonal fiber structure [3]. Literature [4] proposed an approach for the assessment of the distribution of the traction force for a crack with bridging fibers in an infinite, orthotropic elastic plane under a uniform remove tension stress. Most researchers, such as Woo, Lee and Tsai [5-7] etc, almost investigated static problems of composite materials; moreover, they obtained only numerical solutions. Literature [8] set up a model of bridging fiber pull-out, but it also acquired the numerical solutions under the static conditions. It is indispensable to consider the mechanical analysis of matrix cracking with bridging fibers, so as to evaluate the distribution of the axis traction force in each fiber. However, the fractures of composite materials often arise in dynamic conditions, so accordingly it is extremely important to research their fracture dynamics problems. In an orthotrpic medium, elastodynamics crack problems were studied and closed solutions were also gained, but bridging fiber pull-out problems weren't dealt with in literatures $[9,10]$. Bridging fiber pull-out is very complicated and cockamamie in dynamic fracture process of composite materials, so a lot of difficulty must be overcome in studying dynamic crack expansion problems on bridging fiber pull-out of composite materials. When composite materials occur in a crack, bridging fiber pull-out often exists ahead of the crack tips, and this is a frequent phenomenon.

Because the fiber failure is governed by maximum tensile stress, which appears at the crack plane, the fiber breaks and hence the crack propagation should occur in a self-similar fashion. The fiber breaks along a transverse line and therefore present a notch [8,11-12]. When a crack runs at higher velocity, bridging fiber pull-out still exists in the dynamic case of composite materials, which are more important than those in the statics.

The problem under consideration is that of a crack, moving in one plane, presumed to nucleate from an infinitesimally small micro-crack with maximum velocity from the start. This modality of symmetrical crack, running with constant velocity $V$ in both the positive and negative directions of the $x$-axis, has been researched by 
Broberg [13] and Craggs [14]. Both considered motions in materials postulated to be homogeneous and isotropic, as regards stress-strain relationships and fracturing characters. If the fiber failure is governed by maximum tensile stress, which appears at the crack plane, the fiber breaks and hence the crack expansion should occur in the modality of self-similarity $[13,14]$. The fiber breaks along a transverse line and therefore presents a notch [7, 11-12]. When a crack runs at higher speed, bridging fibers still exist in the dynamic situation of composite materials, which are more significant than those in the statics. Since bridging fibers can result in stabilizing effect of crack extension problem along the original notch plane, the dynamic fracture influence on bridging fibers of composite materials will be shown in detail; at the same time, stresses and displacements as well as stress intensity factors are deduced properly.

In this paper, the dynamic expansion problem on an internal central crack with bridging fibers of composite materials is analyzed by the ways of Keldysh-Sedov mixed boundary value problem, and analytical solutions for unidirectional reinforced material with fibers parallel to the free surface are shown. First, the solution of a sole dislocation in an elastically half-plane is derived from the uses of complex variable analysis. The crack is then displayed in terms of a consecutive distribution of dislocation. This solution which has relation to a bridging fiber force induces a system of self-similar functions with dislocation density as unknown quantities. Then self-similar functions are resolved analytically by means of KeldyshSedov's method.

\section{A Dynamic Model of Bridging Fiber Pull-out of Composite Materials}

When fiber-reinforced composite materials occur a crack, phenomenon of bridging fiber pull-out will often occur ahead of the crack tips and off $x$-axis on occasion, and analyzing crack problems in this situation is more difficult. Composite materials are often referred to as orthotropic aniostropic body in virtue of the direction of their fibers, while bridging fibers play an important role in their strength, consequently queries on bridging fiber pull-out are one of the most complex advancing tasks in mechanics of composite materials. When a crack moves with high speed, bridging fiber pull-out phenomenon of composite materials is still likely to exist. Because the problems of bridging fibers are more complicated and cockamamie, there is a lot of difficulty in mathematical calculations. In order to resolve dynamic fracture queries of bridging fiber pull-out of unidirectional composite materials, it is indispensable to establish a suitable symmetrical dynamic model of bridging fiber pull-out, hence fracture dynamics problems of bridging fiber pull-out of composite materials are effectively solved.

\subsection{Characterization of Dynamic Fracture Problems Concerning Bridging Fiber Pull-Out}

The problem of an internal central crack with bridging fiber pull-out of composite materials is analyzed under the dynamic conditions by means of Reimann-Hilbert mixed boundary value, and that analytical solutions for unidirectionally reinforced composite materials with bridging fibers parallel to the free surface are presented. This solution in conjunction with a bridging fiber force gives rise to a system of self-similar functions with dislocation density as unknown units. The self-similar functions are solved analytically using Reimann-Hilbert method. In order to settle efficaciously fracture problems on bridging fibers of composite materials, it is inevitable to establish dynamic models of bridging fibers. Since bridging fibers can conduce a stabilizing effect on crack extension problems along the original notch plane, the dynamic fracture effect of bridging fiber pull-out in composite materials will be shown, at the same time, stresses and displacements as well as stress intensity factors are deduced appropriately. In order to resolve efficiently fracture problems of bridging fiber pull-out of composite materials, proper dynamic models of bridging fiber pullout must be built. Only this approach, can a dynamic crack expansion problem of bridging fiber pull-out of composite materials obtain content solutions.

\subsection{Base of A Dynamic model of Bridging Fibers}

The crack is postulated to nucleate an infinitesimally small micro-crack situated along the $x$-axis in the form of self-similarity with the high speed extension, and to run symmetrically in the positive and negative $x$ directions with the constant crack tip velocity $V$ in the matrix. Bridging fiber pull-out of composite materials discussed is modeled as a two-dimensional region, having a sole row of parallel, same, equally spaced fibers, separated by matrix $[15,16]$. Initial breaks originate from an arbitrary number broken fibers leading to the fiber breaks along a transverse line and therefore present a notch. Furthermore, an arbitrary number of self-similar (off-axis) fiber breaks, i.e. fiber pull-out, with symmetry at the origin of coordinates and along a transverse line are also considered. A schematic of a dynamic model of bridging fiber pull-out configuration is described in Figure 1. Since the configuration in Figure $\mathbf{1}$ is symmetry both in geometry and loading, respectively, with respective to y-axis, only the right half-plane of the zone needs to be considered for analyses. The fibers and the matrix are taken to be linearly elastic. It is further presumed that the fibers have a much higher elastic modulus in the axial direction than the matrix and therefore the fibers are usually taken as supporting all of the axial loadings in composite materi- 


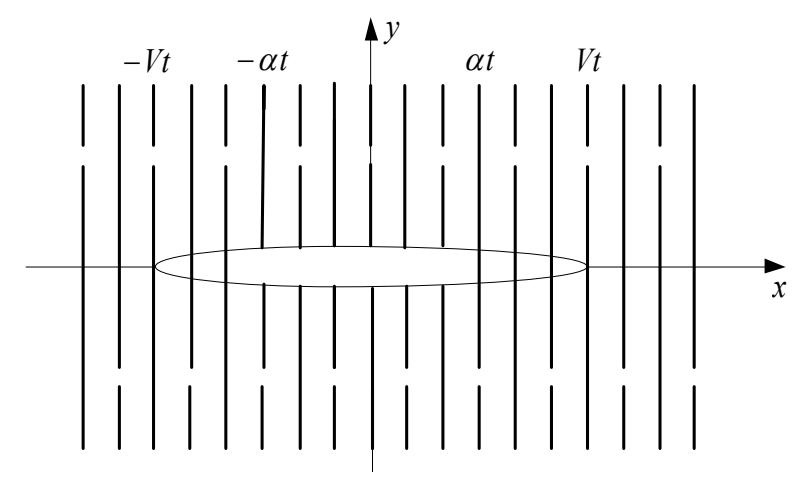

Figure 1. Schematic of a dynamic model of bridging fiber pull-out configuration.

als. Load is transferred between adjacent fibers through the matrix by a straightforward shear mechanism $[15,16]$. The shear stresses are independent of transverse displacements and the equilibrium equation in the fiber direction reduces to an equation in the longitudinal displacements alone, as is a typical of shear-lag theory $[11,12]$. The solution approaches and modeling procedure put forward by [11] will be utilized, which discussed static problems, the fiber fractures in turns occur along two sole planes, i.e. the fiber fracture was self-similar fiber (off-axis) break. By virtue of the point, break lie is same in geometry off $x$-axis, i.e. break is symmetry about the origin of the coordinate. In short, the fiber fracture was self-similar fiber (off-axis) break and presented a notch. Bridging fiber pull-out occur ahead of the crack tips and off $x$-axis. Bridging fibers do not break in the area of the crack tips along the crack plane, but the others fracture off the further of the crack tips, i.e. at the central section of crack or notch. When the crack runs, fibers continuously break with the constant velocity $\alpha$ according to my assumption. As shown in Figure 1, at $y=0$, the realm of crack or notch in matrix is $|x|<V t$; and the fibers broke at the extent of $|x|<\alpha t$; while the zone of bridging fibers is $\alpha t<|x|<V t$, respectively $[15,16]$. At $\mathrm{y} \neq 0$, the bridging pull-out positions locate at $|x|>V t[8]$.

Evidently, the dynamic model of crack extension problem with bridging fiber pull-out in Figure $\mathbf{1}$ is clarified by that in Figure 2. This is a model of symmetrical crack propagation, running with constant velocity $V$ in both the positive and negative directions of $x$-axis, at the same time, bridging fibers fracture with constant velocity $\alpha$. The area of bridging fibers has the symmetrical state with respect to $y$-axis $[15,16]$. Each bridging fiber is replaced by a pair of vertical traction forces which act at the points with the same $x$-coordinate on the upper and lower crack surfaces, but in an opposite direction. Each bridging fiber is postulated to be balanced with the fracture load of a fiber from the matrix. The present model has the symmetries of geometrical and mechanical conditions with respect to the $x$ - and $y$-axes on account of the symmetrical

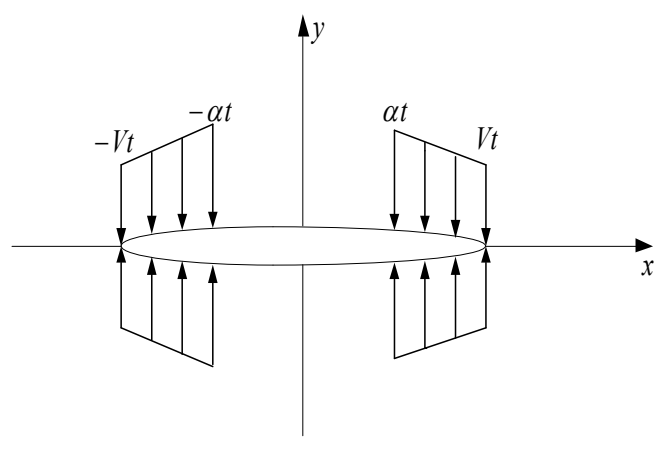

Figure 2. Dynamic model of crack-face bridging fiber zone.

crack expansion. At $y=0$, traction forces act in the zone of $\alpha t<|x|<V t$, which represent fibrous tensile stresses which don't act in the rest of the crack $[15,16]$. Bridging fibers of composite materials are usually arranged tightly, separated by matrix, therefore bridging fiber traction forces are presumed to be distributed consecutively. At $y$ $=0, \alpha t<|x|<V t$, bridging fiber pull-out has symmetry with respective to the origin of the coordinate; the displacements of the crack face are not the same, but traction forces of bridging fibers are identical [15-17]. In short, traction forces of bridging fibers are homogenous in this section, whose magnitude is $P$ assumed. On the other hand, when the crack extends with high speed, the magnitude of crack will increase with time $t$; the longer the crack spreads, the more fibers break. The above analyses are postulated that fibers in matrix are distributed uniformly, and each fiber has the identical strength, moreover, the fracture fibers and matrix simultaneity occur in the same segment of the crack expansion plane [7,15-17]. It is distinct that traction forces are larger near the points of $\pm \alpha t$, and they are smaller close to the points of $\pm V t$. When the crack runs at high speed, its dimension has relation to the parameters $x$ and $t$, and the surfaces of the crack subjected to loads must also be related to $x$ and $t$. When the fracture occurs, both the fiber and the matrix are in the same plane of crack expansion [15-17]. Of course, this is an assumed model which maybe not coincident with that in practicality. We can reasonably interpret this to mean that, outside the crack or notch, conditions are steady-state because the crack had no effect it, after all we can only expect the crack to affect appreciably the part of the body which lies in a certain proximity to it.

\section{Universal Expressions of Electrodynamics Equations for Orthotropic Anisotropy}

In order to solve efficaciously fracture dynamics queries of bridging fibers of composite materials, solutions will be attained under the action of point forces for mode I motive crack. In terms of the theorem of generalized functions, the problems dealt with unlike boundary con- 
ditions will be facilely translated into Reimann-Hilbert mixed boundary value problem by means of self-similar functions, then correlative solutions will be obtained.

Postulate at $y=0$ that there are any number of loaded sections and displacement sections along the $x$-axis, and the ends of these segments are running with unlike constant velocity. At $t=0$, the half-plane is at rest. In these sections the loads and displacements are discretionary linear compages of the following functions [15-22]:

$$
\begin{array}{r}
\frac{\mathrm{d}^{k} f_{k_{1}}(x)}{\mathrm{d} x^{k}} \cdot \frac{\mathrm{d}^{s} f_{s_{1}}(t)}{\mathrm{d} t^{s}} \\
\text { Where } f_{i}(\xi)= \begin{cases}0 & \xi<0 \\
\xi^{i} & \xi>0\end{cases}
\end{array}
$$

Here $k, k_{1}$ and $s, s_{1}$ are discretionary integer positive numbers [15-22].

A discretional successive function of two variables $x$ and $t$ may be shown as a linear superposition of Equation (1), therefore resolving loads or displacements with the form of Equation (2) will possess significance in princple. Introduce the linear differential operator as well as inverse:

$$
L=\frac{\partial^{m+n}}{\partial x^{m} \partial t^{n}}, \text { inverse: } L^{-}=\frac{\partial^{-m-n}}{\partial x^{-m} \partial t^{-n}}
$$

Here $+m+n,-m-n$ and 0 represent the $(m+n)$ th order derivative, the $(m+n)$ th order integral and function's self. It is facile to prove that there exist constants $m$ and $n$, when $L$ is put into Equations (1), (2), homogeneous functions of $x$ and $t$ of zeroth dimension (homogeneous) are gained. The coefficients $m, n$ will be called the indices of self-similarity [15-22].

Using relative expressions of elastodynamics equation of motion for an orthotropic anisotropic body [15-22]:

For the case when function $L v$ is homogeneous $[5,6]$ :

$$
v^{0}=L v, \quad \sigma_{y}^{0}=L \sigma_{y}
$$

For the case when function $L \sigma_{y}$ is homogeneous:

$$
v^{0}=\partial L v / \partial t, \quad \sigma_{y}^{0}=\partial L \sigma_{y} / \partial t
$$

The relative self-similar functions are as [15-23]:

$$
v^{0}=\operatorname{Re} W(\tau), \quad \sigma_{y}^{0}=(1 / t) \operatorname{Re} F(\tau)
$$

Where: $v^{0}$ and $\sigma_{y}^{0}$ in Equations (4)-(6) are the notation in [15-17,20-23], and they are relevant variables $\tau$ and $t$ which directly work out displacements and stresses by the course of respective calculations in Equation (7).

$$
W^{\prime}(\tau)=\left[D_{1}(\tau) / D(\tau)\right] F(\tau)
$$

Where: $\tau=x / t, F(\tau), W(\tau)$ are self-similar functions. The values of $D_{1}(\tau) / D(\tau)$ can be ascertained from Appendix 1 of literatures [15-17,20-22], indicated here are only: $D_{1}(\tau) / D(\tau)$ in the neighborhood of the subsonic speeds is purely imaginary for the considered values. Thus, elastodynamics problems for an orthotropic anisotropic body studied can be changed into seeking the sole unknown function problems of $F(\tau)$ and $W(\tau)$ for which must meet the boundary-value conditions. In the universal situation this is Riemann-Hilbert problem in the theory of complex functions (in the simplest cases we have Keldysh-Sedov or Dirchlet problem), this kind of problem is facilely settled by the usual approaches, for example, in the books by Muskhelishvili [24,25].

Fracture dynamics problems will be investigated for an infinite orthotropic anisotropic body. Assume at the initial moment $t=0$ a crack occurs at the origin of coordinates and begins spreading at constant velocity $V$ (for the subsonic velocities) along the positive direction of $x$-axis; and at $t<0$, the half-plane was at rest. The surfaces of the crack are subjected to the unlike types of loads under the plane strain states.

\section{Fundamental Format of the Solution of Symmetrical Dynamic Extension Query Concerning Mode I Crack}

At the initial moment $t=0$, a micro-crack is supposed to appear in an orthotropic anisotropy. Let the Cartesian coordinate axes align with the axes of elastic symmetry of the body. The problem considered is restricted to motion in the $x-y$-plane. The crack is moving symmetrically with constant velocity $V$ along the positive and negative directions of $x$-axis respectively. The problems will be changed into the following boundary condition queries:

$$
\begin{aligned}
& \sigma_{y}(x, 0, t)=f_{1}(x, t),|x|<V t \\
& v(x, 0, t)=0, \quad|x|>V t
\end{aligned}
$$

Introducing the variable $\tau=x / t$. By means of the above correlative expressions and $t \delta(x)=\delta(x / t)$ in the theory of generalized functions [26-28], the boundary conditions can be transformed as:

$$
\begin{aligned}
& \operatorname{Re} F(\tau)=f_{2}[\tau, \delta(\tau)],|\tau|<V \\
& \operatorname{Re} W^{\prime}(\tau)=0,|\tau|>V
\end{aligned}
$$

In the light of the relationship of $F(\tau)$ and $W(\tau)$ in Equation (7) and the previous conditions, the format of sole unbeknown function $W^{\prime}(\tau)$ can be confirmed:

$$
W^{\prime}(\tau)=f_{3}[\tau, \xi(\tau)]
$$

The problems can reduce to Keldysh-Sedov problem:

$$
\begin{aligned}
& \operatorname{Re} \xi(\tau)=0,|\tau|>V \\
& \operatorname{Im} \xi(\tau)=0,|\tau|<V
\end{aligned}
$$

Considering symmetry and the infinite point of the plane corresponding to the origin of coordinates of the physical plane as well as singularities of the stress at the 
crack tip $[29,30]$, the solution in the above problems can be readily deducted by literatures $[15-16,20]$ as:

$$
\xi(\tau)=T[(V-\tau),(V-\tau)]
$$

Using Equations (6) or (7), we will easily obtain the stress, the displacement and the stress intensity factor under the conditions of mode I crack extension problems.

\section{The Solutions of Practical Problems}

In order to solve symmetrical dynamics problems with bridging fiber pull-out of unidirectional composite materials, solutions will be found under the conditions of different loads for mode I expanding crack. According to the theorem of generalized functions, the unlike boundary condition problems investigated will be translated into Keldysh-Sedov mixed boundary value problem by the approaches of self-similar functions, and the corresponding solutions will be acquired.

1) Suppose at the initial moment $t=0$ a crack occurs at the coordinate origin and begins to spread at constant velocity $V$ in both directions along the $x$-axis. The edges of the crack are subjected to normal point force $P t^{5} / x^{5}$, moving at a constant velocity $\beta$ along the positive direction of $x$-axis, where $\beta<V$; at $t<0$ the half-plane was at rest. The boundary conditions will be as follows:

$$
\begin{aligned}
& \sigma_{y}(x, 0, t)=-P t^{5} / x^{5} \cdot \delta(x-\beta t),|x|<V t \\
& v(x, 0, t)=0,|x|>V t
\end{aligned}
$$

In this case the displacement will distinctly be homogeneous functions, in which $L=1$. Using $\tau=x / t$ and the theory of generalized functions [26-28] and Equations (4) and (6), the first of Equation (13) can be as:

$$
\begin{aligned}
\operatorname{Re} F(\tau) & =-P t^{5} / x^{5} \cdot t \delta(x-\beta t) \\
& =-P \tau^{-5} \delta(\tau-\beta) \quad|\tau|<V
\end{aligned}
$$

In the light of Equation (7), boundary conditions (14) will be further rewritten:

$$
\begin{aligned}
& \operatorname{Re}\left[W^{\prime}(\tau) \frac{D(\tau)}{D_{1}(\tau)}\right]=-P \tau^{-5} \cdot \delta(\tau-\beta),|\tau|<V \\
& \operatorname{Re}\left[W^{\prime}(\tau)\right]=0, \quad|\tau|>V
\end{aligned}
$$

Deducting from the above-mentioned formulae, the unique solution of $W^{\prime}(\tau)$ must have the modality:

$$
W^{\prime}(\tau)=\xi(\tau) /\left[\tau^{5}(\tau-\beta)\right]
$$

$\xi(\tau)$ has no singularity in the domain of $|\tau|<V$, while $D_{1}(\tau) / D(\tau)$ is purely imaginary for the subsonic speeds, consequently $\xi(\tau)$ must be purely real in this segment. Thus, question (15) can conduce the following problems:

$$
\begin{aligned}
& \operatorname{Re} \xi(\tau)=0,|\tau|>V \\
& \operatorname{Im} \xi(\tau)=0,|\tau|<V
\end{aligned}
$$

According to symmetry and the conditions of the infinite point of the plane corresponding to the origin of coordinates of the physical plane as well as singularities of the stress of the crack tip [29,30], the sole solution of the Keldysh-Sedov problem (17) under the given conditions must have the following modality:

$$
\xi(\tau)=A\left(V^{2}-\tau^{2}\right)^{-1 / 2}
$$

where $A$ is an unknown constant.

Inserting Equation (18) into (16), (7), one can gain:

$$
\begin{gathered}
W^{\prime}(\tau)=A /\left[\tau^{5}(\tau-\beta)\left(V^{2}-\tau^{2}\right)^{1 / 2}\right] \\
F(\tau)=\frac{A \cdot D(\tau) / D_{1}(\tau)}{\tau^{5}(\tau-\beta) \sqrt{V^{2}-\tau^{2}}}
\end{gathered}
$$

Then putting Equation (20) into (14), at $\tau \rightarrow \beta$, constant $A$ can be determined from that

$$
A=-P \sqrt{V^{2}-\beta^{2}} /\left\{\pi \cdot \operatorname{Im}\left[D(\beta) / D_{1}(\beta)\right]\right\}
$$

Putting Equation (20) into (6) and (4), at the surface $y$ $=0$, the stress $\sigma_{y}$, the displacement $v$ and the stress intensity factor $K_{1}(t)$ are acquired, respectively:

$$
\begin{gathered}
\sigma_{y}=\operatorname{Im}\left[\frac{D(\tau)}{D_{1}(\tau)}\right] \cdot \frac{A}{\tau^{5}(\tau-\beta) \sqrt{x^{2}-V^{2} t^{2}}},|x|<V t \\
K_{1}(t)=\sqrt{\frac{\pi}{t}} \cdot \frac{A}{V^{11 / 2}(V-\beta)} \cdot \operatorname{Im}\left[\frac{D(V)}{D_{1}(V)}\right]
\end{gathered}
$$

Replacing Equation (19) into (4), (6), after integrating with respective to $\tau$ one can obtain the displacement $v$ :

$$
\begin{gathered}
v=\frac{A}{\beta} \cdot \operatorname{Re} \int_{\infty}^{x / t}\left[-\frac{1}{\beta^{4} \tau}-\frac{1}{\beta^{3} \tau^{2}}-\frac{1}{\beta^{2} \tau^{3}}-\frac{1}{\beta \tau^{4}}\right. \\
\left.-\frac{1}{\tau^{5}}+\frac{1}{\beta^{4}(\tau-\beta)}\right] \frac{1}{\sqrt{V^{2}-\tau^{2}}} \mathrm{~d} \tau
\end{gathered}
$$

Utilizing correlative integral formulas [31] to yield:

$$
\begin{gathered}
\int \frac{1}{\tau \sqrt{V^{2}-\tau^{2}}} \mathrm{~d} \tau=\frac{1}{V} \ln \frac{V-\sqrt{V^{2}-\tau^{2}}}{\tau} \\
\int \frac{1}{\tau^{2} \sqrt{V^{2}-\tau^{2}}} \cdot \mathrm{d} \tau=-\frac{\sqrt{V^{2}-\tau^{2}}}{V^{2} \tau} \\
\int \frac{1}{\tau^{3} \sqrt{V^{2}-\tau^{2}}} \mathrm{~d} \tau=-\frac{\sqrt{V^{2}-\tau^{2}}}{2 V^{2} \tau^{2}}+\frac{1}{2 V^{3}} \ln \frac{V-\sqrt{V^{2}-\tau^{2}}}{\tau} \\
\int \frac{\tau^{-4}}{\sqrt{V^{2}-\tau^{2}}} \mathrm{~d} \tau=-\frac{\sqrt{V^{2}-\tau^{2}}}{3 V^{2} \tau^{3}}-\frac{2 \sqrt{V^{2}-\tau^{2}}}{3 V^{4} \tau}
\end{gathered}
$$




$$
\begin{aligned}
& \int \frac{1}{\tau^{5} \sqrt{V^{2}-\tau^{2}}} \mathrm{~d} \tau=\frac{3}{8 V^{5}} \ln \frac{V-\sqrt{V^{2}-\tau^{2}}}{\tau} \\
& -\frac{1}{4 V^{2}} \frac{\sqrt{V^{2}-\tau^{2}}}{\tau^{4}}-\frac{3}{8 V^{4}} \frac{\sqrt{V^{2}-\tau^{2}}}{\tau^{2}} \\
& \int \frac{1}{(\tau-\beta) \sqrt{V^{2}-\tau^{2}}} \mathrm{~d} \tau \\
& =\frac{1}{\sqrt{V^{2}-\beta^{2}}} \ln \left|\frac{V^{2}-\beta \tau-\sqrt{\left(V^{2}-\beta^{2}\right)\left(V^{2}-\tau^{2}\right)}}{V(\beta-\tau)}\right|
\end{aligned}
$$

The crack extends along the $x$-axis, therefore $W(\tau)$ can be worked out in the operation of the definite integral, we take constant $C=0$. Then putting Equations (25)-(30) into (24), the displacement $v$ is given as follows:

$$
\begin{aligned}
v= & \frac{-A}{\beta V}\left(\frac{1}{\beta^{4}}+\frac{1}{2 \beta^{4} V^{2}}+\frac{3}{8 V^{4}}\right) \ln \left|\frac{V t-\sqrt{V^{2} t^{2}-x^{2}}}{x}\right| \\
& +\frac{A}{\beta^{4} \sqrt{V^{2}-\beta^{2}}} \ln \left|\frac{V^{2} t-\beta x-\sqrt{\left(V^{2}-\beta^{2}\right)\left(V^{2} t^{2}-x^{2}\right)}}{V(\beta t-x)}\right| \\
& +\frac{A}{\beta^{2} V^{2}}\left(\frac{1}{\beta^{2}}+\frac{t}{2 \beta x}+\frac{t^{2}}{3 x^{2}}+\frac{2}{3 V^{2}}+\frac{\beta t^{3}}{4 x^{3}}+\frac{3 \beta t}{8 V^{2} x}\right) \\
& \frac{\sqrt{V^{2} t^{2}-x^{2}}}{x},|x|<V t
\end{aligned}
$$

By means of the solution of Equation (31), the bridging fiber fracture speed $\alpha$ can be readily attained as:

$$
\begin{aligned}
v= & \frac{-A}{\beta V}\left(\frac{1}{\beta^{4}}+\frac{1}{2 \beta^{4} V^{2}}+\frac{3}{8 V^{4}}\right) \ln \left|\frac{V-\sqrt{V^{2}-\alpha^{2}}}{\alpha}\right| \\
& +\frac{A}{\beta^{4} \sqrt{V^{2}-\beta^{2}}} \ln \left|\frac{V^{2}-\beta \alpha-\sqrt{\left(V^{2}-\beta^{2}\right)\left(V^{2}-\alpha^{2}\right)}}{V(\beta-\alpha)}\right| \\
& +\frac{A}{\beta^{2} V^{2}}\left(\frac{1}{\beta^{2}}+\frac{1}{2 \beta \alpha}+\frac{1}{3 \alpha^{2}}+\frac{2}{3 V^{2}}+\frac{\beta}{4 \alpha^{3}}+\frac{3 \beta}{8 V^{2} \alpha}\right) \\
& \frac{\sqrt{V^{2}-\alpha^{2}}}{\alpha},|x|=\alpha t
\end{aligned}
$$

Each fibre has same strength [15-17,20-22] according to assumption, hence the bridging fiber fracture strength must be equal. Where $\Delta$ can be ascertained by an axial tensile test of bridging fibers of composite materials with $V$ and $\beta$ regarded as known constants, respectively.

In terms of this approach, the bridging fiber fracture speed $\alpha$ can be only gained numerical solution, because it can not be shown in the format of explicit function.

2) With all conditions remaining the same as those in the above sample, the applied loads become variable loads $P x^{5} / t^{4}$. The boundary conditions of the problem will be as follows:

$$
\begin{aligned}
& \sigma_{y}(x, 0, t)=-P x^{5} / t^{4} \cdot \delta(x-\beta t),|x|<V t \\
& v(x, 0, t)=0,|x|>V t
\end{aligned}
$$

In this case the stresses are apparently homogeneous functions, in which $L=1$, making use of the theory of generalized functions [26-28] and Equations (5) and (6), the first of the boundary conditions (33) can be rewritten in the following modality:

$$
\begin{aligned}
\operatorname{Re} F(\tau) & =-(-4) P x^{5} / t^{5} \cdot t \delta(x-\beta t) \\
& =4 P \tau^{5} \delta(\tau-\beta) \quad|\tau|<V
\end{aligned}
$$

Owing to the derivative of Dirac's function equaling zero at $x \neq \beta t$, the above expression will be deducted.

In terms of Equation (7), boundary conditions (33) will be further rewritten:

$$
\begin{aligned}
& \operatorname{Re}\left[W^{\prime}(\tau) \frac{D(\tau)}{D_{1}(\tau)}\right]=4 P \tau^{5} \cdot \delta(\tau-\beta),|\tau|<V \\
& \operatorname{Re}\left[W^{\prime}(\tau)\right]=0,|\tau|>V
\end{aligned}
$$

Known from the above, the sole solution of $W^{\prime}(\tau)$ is:

$$
W^{\prime}(\tau)=\xi(\tau) \cdot \tau^{5} /(\tau-\beta)
$$

$\xi(\tau)$ has no singularity in the domain of $|\tau|<V$, while $D_{1}(\tau) / D(\tau)$ is purely imaginary for the subsonic speeds, so $\xi(\tau)$ must be purely real in this area. Thus, question (36) will be the following boundary value problems:

$$
\begin{aligned}
& \operatorname{Re} \xi(\tau)=0,|\tau|>V \\
& \operatorname{Im} \xi(\tau)=0,|\tau|<V
\end{aligned}
$$

In the light of the symmetrical conditions and singularities of the stress as well as the infinite point of the plane corresponding to the coordinate origin of the physical plane, the sole solution of Keldysh-Sedov problem (37) takes the format:

$$
\xi(\tau)=A\left(V^{2}-\tau^{2}\right)^{-3 / 2}
$$

where $A$ is an unknown constant.

Putting Equation (38) into (37), (7), one can attain:

$$
\begin{gathered}
W^{\prime}(\tau)=A \tau^{5} /\left[(\tau-\beta)\left(V^{2}-\tau^{2}\right)^{3 / 2}\right] \\
F(\tau)=\frac{A \cdot \tau^{5} \cdot D(\tau) / D_{1}(\tau)}{(\tau-\beta)\left(V^{2}-\tau^{2}\right)^{3 / 2}}
\end{gathered}
$$

Putting Equation (40) into (35), we have the result: 


$$
\begin{aligned}
& \operatorname{Re}\left[\frac{D(\tau)}{D_{1}(\tau)} \cdot \frac{A \tau^{5}}{(\tau-\beta)\left(V^{2}-\tau^{2}\right)^{3 / 2}}\right] \\
& =\operatorname{Re}\left[\frac{4 P \tau^{5}}{\pi(\tau-\beta)} i\right], \quad|\tau|<V
\end{aligned}
$$

At $\tau \rightarrow \beta$, constant $A$ can be determined from that:

$$
A=\frac{4 P\left(V^{2}-\beta^{2}\right)^{3 / 2}}{\pi \cdot \operatorname{Im}\left[D(\beta) / D_{1}(\beta)\right]}
$$

In an orthotropic isotropic body, the disturbance range of elastic wave can be shown by the circular area of radius $c_{1} t$ and $c_{2} t$. Here $c_{1}$ and $c_{2}$ are the velocities of longitudinal and transverse waves $\left(c_{1}>c_{2}\right)$ of elastic body, respectively. In an orthotropic anisotropic body, the disturbance range of elastic wave is not the circular area and can not exceed threshold value $C_{\mathrm{d}}=\left(C_{11} / \rho\right)^{1 / 2}$ of elastic body, where $C_{11}$ is an elastic constant of materials. At $|x|>C_{\mathrm{d}} t$, with $\operatorname{Im}\left[D_{1}(\tau) / D(\tau)\right]=0$, thus displacements and stresses are zero with the initiate cases; and this shows that disturbance of elastic wave can not exceed $C_{\mathrm{d}} t$.

Then inserting Equation (40) into (6) and (5), at the surface $y=0$, the stress $\sigma_{y}$ and dynamic stress intensity factor $K_{1}(t)$ are gained, respectively:

$$
\begin{gathered}
\sigma_{y}=A \cdot \operatorname{Re} \int_{\infty}^{x / t}-\frac{\tau^{4} \cdot \operatorname{Im}\left[D(\tau) / D_{1}(\tau)\right]}{(\tau-\beta)\left(\tau^{2}-V^{2}\right)^{3 / 2}} \mathrm{~d} \tau,|x|<V t \\
K_{\mathrm{I}}(t)=\sqrt{\pi t} \cdot \frac{A V^{5 / 2}}{(V-\beta)} \cdot \operatorname{Im}\left[\frac{D(V)}{D_{1}(V)}\right]
\end{gathered}
$$

The limit of Equation (44) belongs to the form $0 \cdot \infty$, which should be only changed into the type of $\infty / \infty$, then its result can be worked out by the approach of L'Hospital theorem $[31,32]$. Dynamic stress intensity factor $K_{1}(t)$ slowly augments from zero and even reaches or exceeds fracture toughness of this material, because unique variable $t$ lies in the numerator, and the rest are also referred to as real constants.

Equation (39) can rewrite as follows

$$
\begin{aligned}
& W^{\prime}(\tau)=\frac{A \tau^{5}}{(\tau-\beta)\left(V^{2}-\tau^{2}\right)^{3 / 2}} \\
& =\left[\tau^{4}+\beta \tau^{3}+\beta^{2} \tau^{2}+\beta^{3} \tau+\beta^{4}+\frac{\beta^{5}}{\tau-\beta}\right] \frac{A}{\left(V^{2}-\tau^{2}\right)^{3 / 2}}
\end{aligned}
$$

Integrating Equation (45), one will attain $W(\tau)$. But it has six items, separate denotation is more convenient, Integral formulas are used in literature [31], now assume:

$$
\begin{aligned}
& W_{1}(\tau)=\int \frac{A \tau^{4}}{\left(V^{2}-\tau^{2}\right)^{3 / 2}} \mathrm{~d} \tau \\
& =\frac{A \tau}{2} \sqrt{V^{2}-\tau^{2}}+\frac{A V^{2} \tau}{\sqrt{V^{2}-\tau^{2}}}-\frac{3 A V^{2}}{2} \arcsin \frac{\tau}{V} \\
& W_{2}(\tau)=\int W_{2}^{\prime}(\tau) \mathrm{d} \tau=\beta \int \frac{A \tau^{3}}{\left(V^{2}-\tau^{2}\right)^{3 / 2}} \mathrm{~d} \tau \\
& =A \beta \sqrt{V^{2}-\tau^{2}}+\frac{A \beta V^{2}}{\sqrt{V^{2}-\tau^{2}}} \\
& W_{3}(\tau)=\int W_{3}^{\prime}(\tau) \mathrm{d} \tau=\beta^{2} \int \frac{A \tau^{2}}{\left(V^{2}-\tau^{2}\right)^{3 / 2}} \mathrm{~d} \tau \\
& W_{4}(\tau)=\beta^{3} \int \frac{\tau \beta^{2}\left[\frac{\tau}{\sqrt{V^{2}-\tau^{2}}}-\arcsin \frac{\tau}{V}\right]}{\left(V^{2}-\tau^{2}\right)^{3 / 2}} \mathrm{~d} \tau=\frac{A \beta^{3}}{\sqrt{V^{2}-\tau^{2}}} \\
& W_{5}(\tau)=\beta^{4} \int \frac{A}{\left(V^{2}-\tau^{2}\right)^{3 / 2}} \mathrm{~d} \tau=\frac{A \beta^{4} \tau}{V^{2} \sqrt{V^{2}-\tau^{2}}}
\end{aligned}
$$

Denominator in Equation (45) contains this term $(\tau-\beta)\left(V^{2}-\tau^{2}\right)^{3 / 2}$, calculation will not be preformed in the light of integral formulae, therefore integral format must be translated into integral which can be fulfilled.

For the sake of convenience, we assume: $X=V^{2}-\tau^{2}$

By variable replacement: $\tau_{1}=\tau-\beta$, this term $X$ can be rewritten as follows:

$$
X=V^{2}-\tau^{2}=V^{2}-\beta^{2}-2 \beta \tau_{1}-\tau_{1}^{2}
$$

Known from it, the following relationship in literature [31] is: $a_{1}=V^{2}-\tau^{2}, b_{1}=-2 \beta, c=-1$, $D=4 a_{1} c-b_{1}^{2}=-4 V^{2}$.

Integrating the sixth term of Equation (45) in terms of relevant formulae in literature [31], we will gain $W_{6}(\tau)$ :

$$
\begin{aligned}
W_{6}(\tau)= & \beta^{5} \int \frac{A \mathrm{~d} \tau}{(\tau-\beta) X^{3 / 2}}=A \beta^{5} \int \frac{\mathrm{d} \tau_{1}}{\tau_{1} X^{3 / 2}} \\
= & \frac{A \beta^{5}}{a_{1}}\left[\frac{1}{\sqrt{X}}+\int \frac{\mathrm{d} \tau_{1}}{\tau_{1} \sqrt{X}}-\frac{b_{1}}{2} \int \frac{\mathrm{d} \tau_{1}}{X^{3 / 2}}\right] \\
= & \frac{A \beta^{5}}{a_{1}}\left[\frac{D-b_{1}^{2}-2 b_{1} \beta}{D \sqrt{X}}+\frac{2 b_{1} \tau}{D \sqrt{X}}\right. \\
& \left.-\frac{1}{\sqrt{a_{1}}} \ln \left|\frac{\sqrt{X}+\sqrt{a_{1}}}{\tau-\beta}+\frac{b_{1}}{2 \sqrt{a_{1}}}\right|\right]+C
\end{aligned}
$$

Known from Equation (45):

$W(\tau)=W_{1}(\tau)+W_{2}(\tau)+W_{3}(\tau)+W_{4}(\tau)+W_{5}(\tau)+W_{6}(\tau)$. 
The crack runs along $x$-axis, hence $W(\tau)$ comprising Equations (46)-(50) and (52) can be performed in the definite integral operation, one takes constant $\mathrm{C}=0$. Known from Equation (45):

$W(\tau)=W_{1}(\tau)+W_{2}(\tau)+W_{3}(\tau)+W_{4}(\tau)+W_{5}(\tau)+W_{6}(\tau)$. The crack runs along the $x$-axis, so $W(\tau)$ comprising Equations (46)-(50) and (53) can be performed in the definite integral operation, one takes constant $C=0$.

Making use of relative integral formulas [31] to yield:

$$
\begin{gathered}
\int \frac{1}{\sqrt{V^{2}-\tau^{2}}} \mathrm{~d} \tau=\arcsin \frac{\tau}{V} \\
\int \frac{\sqrt{V^{2}-\tau^{2}}}{\tau} \mathrm{d} \tau=\sqrt{V^{2}-\tau^{2}}+V \ln \frac{V-\sqrt{V^{2}-\tau^{2}}}{\tau} \\
\int \frac{\sqrt{V^{2}-\tau^{2}}}{\tau^{2}} \mathrm{~d} \tau=-\frac{\sqrt{V^{2}-\tau^{2}}}{\tau}-\arcsin \frac{\tau}{V}
\end{gathered}
$$

Putting Equation (46) into (6), (5), the divisional displacement $v_{1}$ will be obtained as:

$$
\begin{aligned}
v_{1}= & -\frac{A x}{2} \cdot \operatorname{Re} \int_{\infty}^{x / t}\left[\frac{1}{\tau} \sqrt{V^{2}-\tau^{2}}+\frac{2 V^{2}}{\tau \sqrt{V^{2}-\tau^{2}}}\right. \\
& \left.-\frac{3 V^{2}}{\tau^{2}} \arcsin \frac{\tau}{V}\right] \mathrm{d} \tau
\end{aligned}
$$

Now putting Equations (54), (26) into (56), there results the divisional displacement $v_{1}$ :

$$
v_{1}=-\frac{A x}{2 t} \cdot \sqrt{V^{2} t^{2}-x^{2}}-\frac{3 A V^{2} t}{2} \cdot \arcsin \frac{x}{V t},|x|<V t
$$

Inserting Equations (47) into (6), (5), by means of Equations (55), (26), the displacement $v_{2}$ are obtained:

$$
v_{2}=A \beta\left[2 \sqrt{V^{2} t^{2}-x^{2}}+x \arcsin \frac{x}{V t}\right],|x|<V t
$$

Replacing Equations (48) into (6), (5), by application of Equation (25), there results the sub-displacement $v_{3}$ :

$$
v_{3}=-A \beta^{2} t \cdot \arcsin \frac{x}{V t}, \quad|x|<V t
$$

Substituting Equation (49) into (6), (5), by means of Equation (26) there results the sub-displacement $v_{4}$ as:

$$
\begin{aligned}
v_{4} & =-\left.A \beta^{3} x \cdot \operatorname{Re}\left(-\frac{\sqrt{V^{2}-\tau^{2}}}{V^{2} \tau}\right)\right|_{\infty} ^{x / t} \\
& =\frac{A \beta^{3}}{V^{2}} \sqrt{V^{2} t^{2}-x^{2}}, \quad|x|<V t
\end{aligned}
$$

Then putting Equation (50) into (6), (5), by means of Equation (25), there results the sub-displacement $v_{5}$ :

$$
v_{5}=-\frac{A \beta^{4} x}{V^{3}} \ln \left|\frac{V t-\sqrt{V^{2} t^{2}-x^{2}}}{x}\right|, \quad|x|<V t
$$

Now substituting Equation (52) into Equations (6), (5), there results the divisional displacement $v_{6}$ as:

$$
\begin{aligned}
v_{6}= & -\frac{A \beta^{5} x}{a_{1}} \operatorname{Re} \int_{\infty}^{x / t} \frac{1}{\tau^{2}}\left[\frac{D-b_{1}^{2}-2 b_{1} \beta}{D \sqrt{X}}\right. \\
& \left.-\frac{1}{\sqrt{a_{1}}} \ln \left|\frac{\sqrt{X}+\sqrt{a_{1}}}{\tau-\beta}+\frac{b_{1}}{2 \sqrt{a_{1}}}\right|+\frac{2 b_{1} \tau}{D \sqrt{X}}\right] \mathrm{d} \tau
\end{aligned}
$$

Integral of the second term of Equation (62) without comprising coefficient can be written as:

$$
\begin{aligned}
& \int-\frac{1}{\tau^{2} \sqrt{a_{1}}} \ln \left|\frac{\sqrt{X}+\sqrt{a_{1}}}{\tau-\beta}+\frac{b_{1}}{2 \sqrt{a_{1}}}\right| \mathrm{d} \tau \\
& =\frac{1}{\sqrt{a_{1}}} \int \ln \left|\frac{\sqrt{X}+\sqrt{a_{1}}}{\tau-\beta}+\frac{b_{1}}{2 \sqrt{a_{1}}}\right| \mathrm{d}\left(\frac{1}{\tau}\right) \\
& =\frac{1}{\tau \sqrt{a_{1}}} \ln \left|\frac{\sqrt{X}+\sqrt{a_{1}}}{\tau-\beta}+\frac{b_{1}}{2 \sqrt{a_{1}}}\right| \\
& -\frac{1}{\beta} \int \frac{1}{\tau} \cdot \frac{\mathrm{d} \tau}{\sqrt{X}}+\frac{1}{\beta} \int \frac{1}{\tau_{1}} \cdot \frac{\mathrm{d} \tau_{1}}{\sqrt{X}}
\end{aligned}
$$

Using integral formulas in Literature [31], one gains:

$$
\int \frac{\mathrm{d} \tau_{1}}{\tau_{1} \sqrt{X}}=-\frac{1}{\sqrt{a_{1}}} \ln \left|\frac{\sqrt{X}+\sqrt{a_{1}}}{\tau_{1}}+\frac{b_{1}}{2 \sqrt{a_{1}}}\right|
$$

where: $a_{1}=V^{2}-\tau^{2}, b_{1}=-2 \beta, c=-1$, $D=4 a_{1} c-b_{1}^{2}=-4 V^{2}$.

Putting Equations (64), (25) into (63), the following representation is given as:

$$
\begin{aligned}
& \int-\frac{1}{\tau^{2} \sqrt{a_{1}}} \ln \left|\frac{\sqrt{X}+\sqrt{a_{1}}}{\tau-\beta}+\frac{b_{1}}{2 \sqrt{a_{1}}}\right| \mathrm{d} \tau \\
& =\left(\frac{1}{\tau}-\frac{1}{\beta}\right) \frac{1}{\sqrt{a_{1}}} \ln \left|\frac{\sqrt{X}+\sqrt{a_{1}}}{\tau-\beta}+\frac{b_{1}}{2 \sqrt{a_{1}}}\right| \\
& -\frac{1}{\beta V} \ln \frac{V-\sqrt{V^{2}-\tau^{2}}}{\tau}
\end{aligned}
$$

Inserting Equations (65), (26) into (62), the divisional displacement $v_{7}$ will be obtained as follows:

$$
\begin{aligned}
v_{6}= & \frac{A \beta^{5}}{V^{2} a_{1}} \cdot \sqrt{V^{2} t^{2}-x^{2}}+\frac{A \beta^{4} x}{V^{3}} \ln \left|\frac{V t-\sqrt{V^{2} t^{2}-x^{2}}}{x}\right| \\
& -\frac{A \beta^{5}}{a_{1}^{3 / 2}}\left(t-\frac{x}{\beta}\right) \ln \left|\frac{\sqrt{V^{2} t^{2}-x^{2}}+t \sqrt{a_{1}}}{x-\beta t}+\frac{b_{1}}{2 \sqrt{a_{1}}}\right||x|<V t
\end{aligned}
$$

The displacement $v$ is the sum of divisional displacement: $v=v_{1}+v_{2}+v_{3}+v_{4}+v_{5}+v_{6}$. The addition of Equations (57)-(61) and (66), the displacement $v$ is acquired as: 


$$
\begin{aligned}
v= & \frac{-A \beta^{5}}{a_{1}^{3 / 2}}\left(t-\frac{x}{\beta}\right) \ln \left|\frac{\sqrt{V^{2} t^{2}-x^{2}}+t \sqrt{a_{1}}}{x-\beta t}+\frac{b_{1}}{2 \sqrt{a_{1}}}\right| \\
& +A\left(\beta x-\frac{3 V^{2} t}{2}-\beta^{2} t\right) \cdot \arcsin \frac{x}{V t} \\
& +A\left(2 \beta+\frac{\beta^{3}}{a_{1}}-\frac{x}{2 t}\right) \sqrt{V^{2} t^{2}-x^{2}},|x|<V t
\end{aligned}
$$

where: $a_{1}=V^{2}-\beta^{2}, b_{1}=-2 \beta, c=-1$, $D=4 a_{1} c-b_{1}^{2}=-4 V^{2}$.

Using the similar ways as that for finding Equation (33), put $|x|=\alpha t$ into (67) while regarding $V, \beta$ and $t$ as known constants, respectively. Bridging fiber fracture speed $\alpha$ can be only gained numerical solution, because it can also not be shown in the form of explicit function.

\section{Law of Dynamic Stress Intensity Factor}

In the light of practical situations of concrete problems, mutative law of dynamic stress intensity factor should be interpreted better. The corresponding parameters are put into Equations (23), (44) to plot $K_{1}(t)$ as a function of time $t$, and their numerical solutions are facilely gained. The following constants are as [8,17,21-22,33-36]:

$C_{11}=19.24 \mathrm{GPa} ; C_{12}=1.25 \mathrm{GPa} ; C_{11}=17.83 \mathrm{GPa} ; P$ $=200 \mathrm{~N} ; C_{66}=1.00 \mathrm{GPa} ; V=300 \mathrm{~m} / \mathrm{s} ; \beta=200 \mathrm{~m} / \mathrm{s} ; \rho=$ $4.9 \times 1000 \mathrm{~N} / \mathrm{m}^{3}$.

Known from Equation (23), dynamic stress intensity factor $K_{1}(t)$ reduces tardily and has obvious singularity on account of unique variable $t$ in the denominator, and the rest units are regarded as real constants. Such a tend is shown in Figure 3. This variable current is similar to the result of Literatures $[8,17,21,22,33-38]$. It is known from Equation (44) that dynamic stress intensity factor $K_{1}(t)$ increases slowly from zero and even reaches or overruns fracture toughness of this material, because sole variable $t$ lies in its numerator, while the rest quantities are also referred to as real constants. This result must conduce the structural instability, as shown in Figure 4. This trend is similar to the result in references $[8,17$, $21,22,33-36,38-40]$, consequently it is also right.

The relative numerical values between dynamic stress intensity factor $K_{1}(t)$ and time $t$ are expressed in Tables $\mathbf{1}$, 2 in terms of curves in Figures 3, 4, respectively.

\section{Conclusions}

By means of correlative expression:

$f(x, y, t)=t^{n} f(x / t, y / t)$, where $n$ is an integral number, the problem considered can be facilely changed into homogeneous function of $x$ and $t$ of zeroth dimension, i.e. self-similar functions. All suffice the relationship of this function, and thus the analytical solutions can be gained by Equations (4)-(7) with homogeneous function of

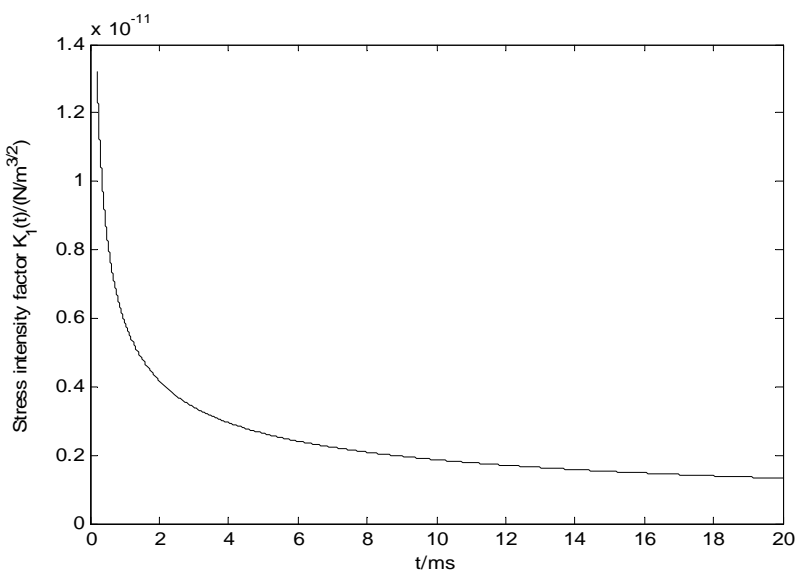

Figure 3. Dynamic stress intensity factor $K_{1}(t)$ versus time $t$.

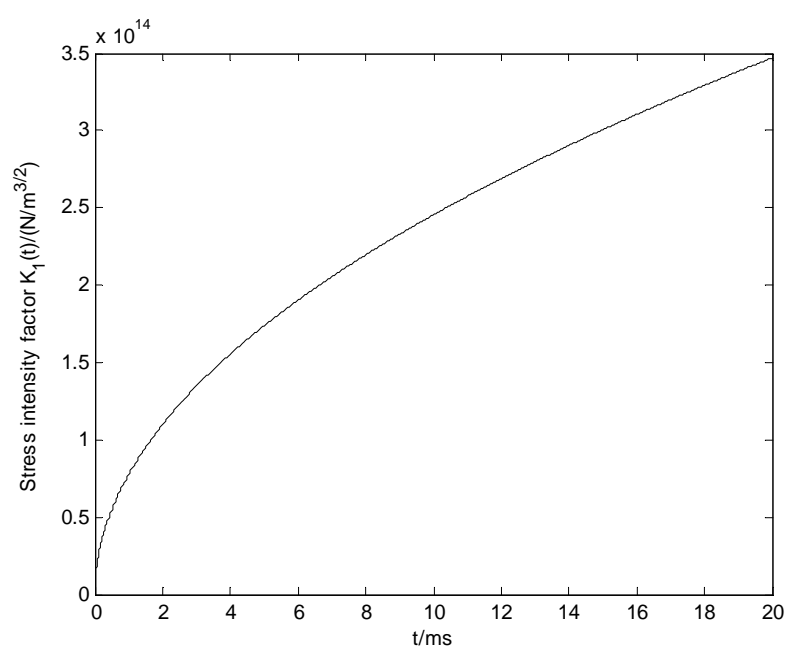

Figure 4. Dynamic stress intensity factor $K_{1}(t)$ versus time $t$.

Table 1. Relative numerical values between $K_{1}(t)$ versus $t$.

\begin{tabular}{cccccc}
\hline$t / \mathrm{ms}$ & 4 & 8 & 12 & 16 & 20 \\
\hline$K_{1}(t)$ & 2.9552 & 2.0896 & 1.7062 & 1.4776 & 1.3216 \\
\hline
\end{tabular}

Table 2. Relative numerical values between $K_{1}(t)$ versus $t$.

\begin{tabular}{cccccc}
\hline$t / \mathrm{ms}$ & 4 & 8 & 12 & 16 & 20 \\
\hline$K_{1}(t)$ & 1.5511 & 2.1936 & 2.6866 & 3.1023 & 3.4684 \\
\hline
\end{tabular}

variable $\tau$. This measure can use not only in electrodynamics [15-20,22,33-34], but also in electrostatics [24,30] and even in other situations, referring to literatures $[30$, 41].

Analytical solutions of the symmetrical dynamic crack extension model for bridging pull-out of unidirectional composite materials were found by way of the theoretical usage of a complex variable function. The method developed in this paper based on the approaches of the selfsimilar functions makes it conceivable to obtain the con- 
crete solution of this model and bridging fiber fracture speed $\alpha$. According to the concrete boundary conditions, self-similar function $W(\tau)$ can be easily deducted by means of corresponding to variable $\tau$. Consequently, analytical solutions of stresses, displacements and dynamic stress intensity factors will be readily worked out. This is referred to as the analogous class of dynamic problem of the elasticity theory. However, the present solution occurs to be the simplest and intuitive of all alternative approaches appearing by so far. Indeed, we have succeeded in a mixed Keldysh-Sedov boundary value problem on a half-plane. The query is of adequate actual interest, since all the members of structures in which fractures may move are of finite dimensions and are frequently in the modality of long strips. The ways of solution are based exclusively on techniques of analytical-function theory and are simple and compendious. By making some observations regarding the solution of the mixed boundary value problem we have rather decreased the amount of the computative work needed to resolve such a crack expansion query.

\section{Acknowledgements (Heading 5)}

Project is supported by the open foundation of the engineering centre of hydraulic and equipment for mining of Liaoning province of China (No. CMHT-201207).

\section{REFERENCES}

[1] D. B. Marshall, B .N. Cox and A .G. Evens, "The Mechanics of Matrix Cracking in Brittle-Matrix Fiber Composites," Acta Metallurgical, Vol. 33, No. 11, 1985, pp. 2013-2021.

http://dx.doi.org/10.1016/0001-6160(85)90124-5

[2] B. Budiansky, J. W. Hutchinson and A. G. Evens, "Matrix Fracture in Fiber-Reinforced Ceramics," Journal of the Mechanics and Physics of Solids. Vol. 34, No. 2, 1986, pp. 167-189.

http://dx.doi.org/10.1016/0022-5096(86)90035-9

[3] M. Ji and H. Ishikawa, "Analysis of an Internal Central Crack with Bridging Fibers in a Finite Orthotropic Plate," International Journal of Engineering Science, Vol. 35, No. 4, 1997, pp. 549-560. http://dx.doi.org/10.1016/S0020-7225(96)00099-7

[4] D. B. Marshall and B. N. Cox. "Tensile Fracture of Brittle Matrix Composites: Influence of Fiber Strength," Acta Metallurgical, Vol. 35, No. 11, 1987, pp. 2607-2619. http://dx.doi.org/10.1016/0001-6160(87)90260-4

[5] Z.-M. Wang, "Mechanics and Structural Mechanics of Composite Materials," Publisher of Machinery Industry, Beijing, 1991.

[6] G.-L. Shen. "Mechanics of Composite Materials," Tsinghua University Press, Beijing, 1996.

[7] C. W. Woo and Y. H. Wang, "Analysis of an Internal Crack in a Fine Anisotropic Plate," International Journal of Fracture, Vol. 62, No. 2, 1993, pp. 203-208.
[8] J. C. Lee, "Analysis of Fiber Bridged Crack near a Free Surface in Ceramic Matrix Composites," Engineering Fracture Mechanics, Vol. 37, No. 2, 1990, pp. 209-219. http://dx.doi.org/10.1016/0013-7944(90)90344-G

[9] W. T. Tsai and I. R. Dharani, "Non Self-Similar Fiber Fracture in Unidirectional Composites," Engineering Fracture Mechanics, Vol. 44, No. 1, 1993, pp. 43-49. http://dx.doi.org/10.1016/0013-7944(93)90080-C

[10] W. N. Liu, "Stress Ahead of the Tip of a Finite-Width Center-Crack in Fiber-Reinforced Composite Specimens: Subjected to Non-Linearly Distributed Bridging Stresses," International Journal of Fracture, Vol. 70, No. 1, 1994, pp. 31-35.

[11] K. Liao and K. Reifsnider, "A Tensile Strength Model for Unidirectional Fiber-Reinforced Brittle Matrix Composite," International Journal of Fracture, Vol. 106, No. 1, 2000, pp. 95-115. http://dx.doi.org/10.1023/A:1007645817753

[12] V. Tamuzs, S. Tarasovs and U. Vilks, "Progressive Delamination and Fibre Bridging Modeling in Double Cantilever Beam Composite Specimens," Engineering Fracture Mechanics, Vol. 68, No. 5, 2001, pp. 513-525. http://dx.doi.org/10.1016/S0013-7944(00)00131-4

[13] A. Piva and E. Viola, "Crack Propagation in an Orthotropic Media", Engineering Fracture Mechanics, Vol. 29, No. 5, 1988, pp. 535-547. http://dx.doi.org/10.1016/0013-7944(88)90179-8

[14] J. De and B. Patra, "Elastodynimic Crack Problems in An Orthotrpic Medium through Complex Variable Approach," Engineering Fracture Mechanics, Vol. 41, No. 5, 1998, pp. 895-909.

[15] K. B. Broberg, "The Propagation of a Brittle Crack," Arkve Fysik, Vol. 18, No. 2, 1960, pp. 159-192.

[16] Y. W. Craggs, "The Growth of a Disk-Shaped Crack," International Journal of Engineering Science, Vol. 4, No. 2, 1966, pp. 113-124. http://dx.doi.org/10.1016/0020-7225(66)90019-X

[17] J. G. Goree and R. S. Gross, "Analysis of a Unidirectional Composite Containing Broken Fibers and Matrix Damage," Engineering Fracture Mechanics, Vol. 33, No. 3, 1979 , pp. 555-578.

[18] G. P. Cherepanov and E. F. Afanasov, "Some Dynamic Problems of the Theory of Elasticity-A Review," International Journal of Engineering Science, Vol. 12, No. 8, 1974, pp. 665-690.

http://dx.doi.org/10.1016/0020-7225(74)90043-3

[19] G. P. Charepanov, "Mechanics of Brittle Fracture," Nauka, Moscow, 1973.

[20] C. Atkinson, "The Propagation of a Brittle Crack in Anistropic Material," International Journal of Engineering Science, Vol. 3, No. 1, 1965, pp. 77-91. http://dx.doi.org/10.1016/0020-7225(65)90021-2

[21] N.-C. Lü, X.-G. Li, Y.-H. Cheng and J. Cheng, "Fracture Dynamics Problem on Mode I Semi-Infinite Crack," Archive of Applied Mechanics, Vol. 81, No. 9, 2011, pp. 1181-1193. http://dx.doi.org/10.1007/s00419-010-0476-x

[22] N. C. Lü, Y. H. Cheng. X. G. Li and J. Cheng, "Dynamic Propagation Problem of Mode I Semi-Infinite Crack 
Subjected to Superimpose Loads," Fatigue \& Fracture of Engineering Materials \& Structures. Vol. 33, No. 3, 2010, pp. 141-148.

[23] N. C. Lü, Y. H. Cheng. X. G. Li and J. Cheng, "An Asymmetrical Dynamic Model for Bridging Fiber PullOut of Unidirectional Composite Materials," Meccanica, Vol. 47, No. 5, 2012, pp. 1247-1260. http://dx.doi.org/10.1007/s11012-011-9509-y

[24] N. I. Muskhlishvili, "Singular Integral Equations," Nauka, Moscow, 1968.

[25] N. I. Muskhlishvili, "Some Fundamental Problems in the Mathematical Theory of Elasticity," Nauka, Moscow, 1966.

[26] F. D. Gakhov, "Boundary-Value Problems," Fitzmatigiz, Moscow, 1963.

[27] R. F. Hoskins, "Generalized Functions," Ellis Horwood, Chichester, 1979.

[28] X. S. Wang, "Singular Functions and Their Applications in Mechanics," Scientific Press, Beijing, 1993.

[29] G. C. Sih, "Mechanics of Fracture 4. Elastodynamics Crack Problems," Noordhoff, Leyden, 1977.

[30] R. P. Kanwal and D. L. Sharma, "Singularity Methods for Eastostatics," Journal of Elasticity, Vol. 6, No. 4, 1976, pp. 405-418. http://dx.doi.org/10.1007/BF00040900

[31] Editorial Group of Mathematics Handbook, "Mathematical Handbook," Advanced Education Press, Beijing, 2002.

[32] Teaching Office of Mathematics of Tongji University. "Advanced Mathematics," Advanced Education Press, Beijing, 1994.

[33] K. C. Wu, "Dynamic Crack Growth in Anisotropic Material," International Journal of Fracture, Vol. 106, No. 1, 2000, pp. 1-12. http://dx.doi.org/10.1023/A:1007621500585

[34] X.-G. Li, Y.-H. Cheng, N.-C. Lv, G.-D. Hao and J. Cheng, "A Dynamic Asymmetrical Crack Model of Bridging Fiber Pull-Out in Unidirectional Composite Materials,"
Journal of Mechanical Science and Technology, Vol. 25, No. 9, 2011, pp. 2297-2309.

http://dx.doi.org/10.1007/s12206-011-0526-5

[35] N. C. Lv, Y. H. Cheng. H. L. Si and J. Cheng, "Dynamics of Asymmetrical Crack Propagation in Composite Materials," Theoretical and Applied Fracture Mechanics, Vol. 47, No. 3, 2007, pp. 260-273. http://dx.doi.org/10.1016/j.tafmec.2007.01.004

[36] N. C. Lü, Y. H. Cheng and J. Cheng, "Mode I Crack Tips Propagating at Different Speeds under Differential Surface Tractions," Theoretical and Applied Fracture Mechanics, Vol. 46, No. 3, 2006, pp. 262-275. http://dx.doi.org/10.1016/j.tafmec.2006.09.004

[37] A. S. Kobayashi, "Dynamic Fracture Analysis by Dynamic Finite Element Method. Generation and Prediction Analyses," In: Nonlinear and Dynarnic Fracture Mechanics, American Society of Mechanical Engineers, New York, 1979, pp. 19-36.

[38] K. Ravi-Chandar and W. G. Knauss, "An Experimental Investigation into Dynamic Fracture: Part 1, Crack Initiation and Arrest," International Journal of Fracture, Vol. 25, No. 41, 1984, pp. 247-262. http://dx.doi.org/10.1007/BF00963460

[39] K. Ravi-Chandar and W. G. Knauss, "An Experimental Investigation into Dynamic Fracture: Part 2, Microstructural Aspects," International Journal of Fracture, Vol. 26, No. 11, 1984, pp. 65-80. http://dx.doi.org/10.1007/BF01152313

[40] K. Ravi-Chandar and W. G. Knauss, "An Experimental Investigation into Dynamic Fracture: Part 3, on SteadyState Crack Propagation and Crack Branching," International Journal of Fracture, Vol. 26, No. 2, 1984, pp. 141152. http://dx.doi.org/10.1007/BF01157550

[41] L. A. Galin, "Contact Problems in Elasticity Theory," GITTL, Moscow, 1953. 